\title{
Incorporation of Dance Practice into the Lifestyle of People with Disabilities and Its Impact on Quality of Life Over Time
}

\begin{abstract}
DeJ esus BM" ${ }^{*}$, Passos $\mathrm{AA}^{2}$, Menezes EO ${ }^{3}$, Adorno $E^{4}{ }^{4}$, DeSantana $J^{M^{5}}$ and Teixeira-Machado $L^{6}$ ${ }^{1}$ Federal University of Sergipe, Graduate Program in Physiological Sciences, Sao Cristovao-SE, Brazil

${ }^{2}$ Federal University of Sergipe, Graduate Program in Applied Health Sciences, Lagarto-SE, Brazil

${ }^{3}$ Federal University of Sergipe, Graduate Program in Health Science, Sao Cristovao-SE, Brazil

${ }^{4}$ Physiotherapist, Federal University of Sergipe, LagartoSE, Brazil

${ }^{5}$ Department of Physical Therapy, Federal University of Sergipe, Graduate Program in Physiological Sciences, Graduate Program in Health Science, Sao Cristovao-SE, Brazil

${ }^{6}$ Department of Education in Health, Graduate Program in Applied Health Sciences, Graduate Program in

National Network for Teaching Environmental Sciences, Federal University of Sergipe, Lagarto-SE, Brazil

*Corresponding author: Beatriz Menezes de J esus, Department of Physiology, Federal University of Sergipe, Av. Marechal Rondon, s/n, J d. Rosa Elze, Sao Cristovao/ SE, Brazil
\end{abstract}

Received: February 23, 2021; Accepted: March 22, 2021; Published: March 29, 2021

\begin{abstract}
Aim: To evaluate how incorporating dance practice into the lifestyle can impact the Quality of Life (QOL) of People with Disabilities (PWD) over four years.

Method: This is a prospective study with dance protocol classes specific for PWD, once a week, 60 min per class, over four years. In addition, the participants performed public presentations each year. Participants were assessed twice per year (at the beginning and at the end of each year of dance practice), totalizing 8 assessments, using the Medical Outcomes Study Survey Short Form 36 (SF36) questionnaire.
\end{abstract}

Results: Forty-one PWD with different clinical conditions, both sexes, ranging in age from 3 to 39 years, participated in the study over four years ( $Y 1$ to Y4). With regard to QOL domains in the SF-36, significant changes were found when comparing the years (two-way ANOVA $(F=19.87 ; p<0.0001)$ ). Values increased in the following QOL domains: Year I: functional capacity, physical aspects, emotional aspects, vitality, pain and mental health. Year II: physical and emotional aspects, pain and mental health. Year III: functional capacity, physical and emotional aspects, vitality, general health, pain and mental health. In Year IV the values increased in all domains.

Interpretation: Over the course of four years, dance practice contributed to the QOL of participants; this suggests that it is an activity that promotes inclusion for PWD in the sociocultural arena.

Keywords: Dance; Persons with disabilities; Lifestyle; Quality of life

\section{Abbreviations}

BMI: Body Mass Index; CP: Cerebral Palsy; FICF: Free and Informed Consent Form; ICD: International Classification of Diseases; IUT: Image Use Term; PWD: Persons with Disabilities; QOL: Quality of Life; ROM: Range of Motion; SEM: Standard Error of Mean; SF-36: Medical Outcomes Study Survey Short Form 36; UN: United Nations; WHO: World Health Organization

\section{Introduction}

According to United Nations (UN) (2006) [1], Persons with Disabilities (PWD) include those who have physical, mental, intellectual or sensory impairments that can obstruct full and effective participation in society with other people. These factors can have a direct influence on Quality of Life (QOL) because they interfere with daily activities and social relationships as well as having an impact on individuals' financial situation. To this end, it is necessary to analyze the QOL levels of PWD [2].

The World Health Organization (WHO) (1995) [3] defines QOL as an individual's perception of their involvement in daily life, in their sociocultural context and the value systems in which they live in relation to their goals, expectations, standards and concerns [4].
A person's entire context can impact QOL, in terms of both physical and mental aspects, including their relationship with the self, the environment and the other.

Evidence has shown that regular practice of physical activities greatly impacts QOL [5]. Some studies suggest that arts, especially dance, also increase QOL in several clinical conditions $[6,7,8]$. A systematic review conducted by Lopez-Ortiz et al. (2018) [9] stated that dance is beneficial for people with Cerebral Palsy (CP); it is associated with balance, gait and cardiorespiratory fitness which have a potential positive impact on emotional well-being.

There are studies showing how dance can improve QOL in different clinical conditions such as: cerebral palsy [9], Parkinson's disease [7], fibromyalgia [10], schizophrenia [11], among others. However, all of these studies used protocols that were applied over a maximum period of 12 months. This raised a question regarding the impact of dance practice on the QOL of PWD over a longer period and in different clinical conditions. Thus, this study hypothesised that dance practice, when incorporates into daily life, can impact QOL in PWD over 48 months of intervention. The aim was to evaluate the impact of dance practice on the Quality of Life (QOL) of PWD over four years.
Phys Med Rehabil Int - Volume 8 Issue 2 - 2021

ISSN : 2471-0377 | www.austinpublishing group.com

DeJesus et al. (c) All rights are reserved
Citation: DeJesus BM, Passos AA, Menezes EO, Adorno ET, DeSantana JM and Teixeira-Machado L. Incorporation of Dance Practice into the Lifestyle of People with Disabilities and Its Impact on Quality of Life Over Time. Phys Med Rehabil Int. 2021; 8(2): 1178. 


\section{Method}

\section{Study design and ethical considerations}

This is a longitudinal interventional study over a period of 48 months, case series type. The Ethics and Research Committee of the Federal University of Sergipe approved this study. All participants and/or caregivers signed Free and Informed Consent Form (FICF) and Image Use Term (IUT) (Appendices 1 and 2).

\section{Participants}

Participants were PWD with different clinical conditions, intellectual impairments and several motor dysfunctions, selected for convenience and recruited at the Ambulatory University, without restrictions of age, sex, dysfunction or limitation restrictions. The exclusion criteria were contraindications for physical activity, severe psychiatric disorder.

\section{Study protocol}

All participants received dance classes specific for PWD called TALT $^{*}$ [12], based on Dalcroze, Feldenkrais and Laban/Bartenieff methods. Dance classes were carried out through this specific protocol, using songs and performances that stimulate imaginary play, essential for the development of communication and social reciprocity. Dance classes occurred in an appropriate space and floor, with a stereo, mirrors and parallel bars, once a week, lasting 60 minutes, for a period of four years. Dance classes consisted in warm-up, group activities, choreographic assemblies and relaxation. In addition, created choreographies were publicly presented in theaters, convention centers, schools, public squares, among others. These presentations occurred twice to three times each year. Within the composition of the classes, activities were divided into three guiding components: the body, the environment, the other(s); and four organizing components: kinesthetic empathy, body resonance, attunement, and synchrony. See details in (Figure 1).

The guiding components address primary factors for promoting functionality at the expense of installed losses due to specificity of each clinical condition.

The Body: activities that make up this axis are based on body image (mental representation that covers space-time organization, associated by affective, cognitive and social aspects); Range of Motion (ROM) (represented by planes and axes of movement for body use in all its permitted angular ranges); motor coordination (bilateral interactions implicit in visuomotor and audiomotor movements); and ability and agility of movements (execution of varied movements bilaterally, exploring mechanisms of motor coordination and body and postural stability) [12-14]. These activities encourage participants to perform and express themselves regardless of their physical condition.

The Environment: the classroom is an essential component for participants' performance during dance, with appropriate space and floor, mirror and sound system. Due to sensory impairments of the PWD (such as auditory and visual hypersensitivity), the room did not contain much sensory information, such as noises, vivid colors on walls and pictures, lamps, among other devices that can draw a lot of attention from participants. In order to activate components of body image and space-time organization, range of motion, motor
(TALT) Dance Protocol

\section{Guiding Components}

\begin{tabular}{|c|c|}
\hline The Body & $\begin{array}{c}\text { The } \\
\text { Environment }\end{array}$ \\
\hline The Other \\
\hline $\begin{array}{c}\text { Kinganizing Components } \\
\text { empathy }\end{array}$ \\
\hline Attunement & Body resonance \\
\hline
\end{tabular}

Figure 1: Systematization of the specific dance protocol for PWD.

coordination and agility of movements, the composition of the classes was based on: (1) planar levels (low, medium and high), (2) movement factors (space (long/short), time (fast/low), fluency (continuous/cut) and weight (light/heavy), (3) directions - displacements during dance steps occurred in all directions (front-back, side-side, and diagonals), and (4) use of devices and props that expanded communication in dance, such as tambourines, rattles, fabric $[13,15,16]$. Diversity of possibilities for movement and space-time perception is presented.

The Other (s): Participants performed dance movements arranged in a circle or in duo that enabled interpersonal experiences and intercorporeal dialogue. Attitude incorporated during dance duets was taken as an example of active non-verbal attunement between partners within performance interaction. Choreographic assemblies triggered a sense of intersubjectivity and differentiation of the self and the other, through structures of shared and synchronized movement. Public presentations reinforced the nature of a subject being in a world with others and being perceived as a center of expectation for others who have not lived through their experiences during dance classes. This fact contributed to important properties of engagement and motivation, which were taken as a phenomenon of two sides between the individual and the other(s). This phenomenon is able to influence social being in a context in which they are mostly on the sidelines, unaware that their actions can be reflected in others the participants were able to perceive it through the eloquent applause of audience, when they saw themselves as central actors, with a positive character, and this predominantly impacted their self-esteem.

To contribute to the important properties of engagement and motivation, we propose four organizational components that can be taken as a phenomenon of two sides between the body and other or others. These greatly influence the involvement of the social being in a given context.

Kinesthetic empathy: imitation by mirroring qualities of movement and shapes as specified in Laban's Movement Analysis, by the planar levels (low, medium and high - squat, jump, rotate sequences; and also the activation of body listening through four movement factors: space (long/short), time (fast/slow), fluency (continuous/cut) and weight (light/heavy). 
Medical Outcomes Study Survey Short Form 36 (SF-36) application

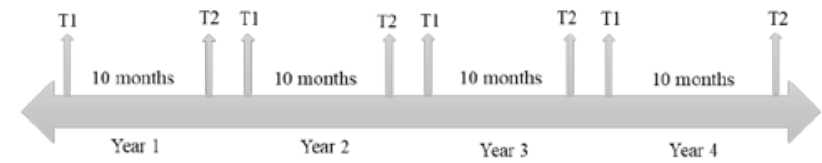

Figure 2: Timeline of the study.

Planar levels and movement factors activate body resonance: this is the adjustment of two bodies moment-to-moment through a bilateral process during dance performance, whether face-to-face, whether bodies are reflected in the mirror or all bodies are on a stage in front of an attentive audience.

Attunement: establishing forms of intersubjectivity, which is the incorporation of body expression that dances for itself or for others, or with others, together, synchronized or not; the attunement to music with what they really want to express; the tuning of the props on stage, the other, and the environment.

Synchrony: coordinating the movement of two or more people, whether in the classroom or in a public presentation. The PWD is mostly on the sidelines, unaware that his actions can be reflected in the other - this can be perceived by the eloquent applause of the audience, when he sees himself as a central actor, with a positive character, impacting predominantly on self-esteem.

\section{Assessment and re-assessment}

Over the four-year period, there were two evaluations per year, one at the beginning and one at the end of each year after the dance protocol, totalizing 8 assessments. To assess QOL, the SF-36 quality of life questionnaire (Medical Outcomes Study Survey Short Form 36SF-36) was applied $[17,18]$. Through this questionnaire it is possible to observe how the individual is characterized according to eight domains: functional capacity, limitation due to physical, social and emotional aspects, vitality, general health, pain and mental health. Each SF-36 question and item has a specific score, which was used for analysis following the calculation of each domain. Minimum value obtained in percentage is zero and maximum is 100 , the higher the score, the greater the relationship of a given domain to an individual's QOL $[17,18]$. Based on this, an analysis of the QOL of PWD was carried out over four years of dance practice. Figure 2 describes the timepoints of assessment (T1 and T2) in each year (Year 1, Year 2, Year 3, Year 4).

\section{Statistical analysis}

Initially data were collected and tabulated in Microsoft Excel, later they were analyzed using Graphpad Prism software version 6.0. All data were tested for normality using the Shapiro Wilk test. Data showing normal distribution were analyzed using a paired T test, and for nonparametric data we used the Wilcoxon test. The Kruskal-Wallis test was used to compare years. Pearson's test for parametric data and the Spearman test for nonparametric data were used for correlation analyzes between domains over the years. The missing data of those participants who gave up or did not complete the assessment were excluded from the study's statistical analysis.

\begin{tabular}{|c|c|c|}
\hline \multicolumn{3}{|c|}{ Sociodemographic Data } \\
\hline \multicolumn{3}{|c|}{$\mathrm{N}=41$} \\
\hline & Age (Years) & $18.37(3-39)$ \\
\hline & Height $(\mathrm{Cm})$ & $136.00(100-160)$ \\
\hline & Weight (Kg) & $37.43(14.5-58)$ \\
\hline & BMI (Kg/m2) & $20.22(10-43.8)$ \\
\hline \multicolumn{3}{|c|}{ Diagnostic Profile } \\
\hline \multicolumn{3}{|c|}{$I C D$} \\
\hline F71 & Moderate intelectual disability & 1 \\
\hline F72 & Severe intelectual disability & 1 \\
\hline F84.0 & Infantile Autism & 12 \\
\hline G11 & Ataxia & 1 \\
\hline G37 & Leukodystrophy & 1 \\
\hline G40 & Epilepsy & 3 \\
\hline G47 & Sleep disturbance & 1 \\
\hline G71 & Muscular distrophy & 2 \\
\hline G80 & Cerebral palsy & 12 \\
\hline G91 & Hydrocephalus & 3 \\
\hline Q90 & Down's syndrome & 4 \\
\hline
\end{tabular}

Note: Values expressed as mean (range). ICD: International Classification of Diseases; BMI: Body Mass Index.

\section{Results}

Over the course of four years, 41 PWD with different clinical conditions participated in this study. These conditions included: ataxia, autism spectrum disorder, cerebral palsy, epilepsy, hydrocephalus, leukodystrophy, moderate and severe intellectual disability, muscular dystrophy, sleep disorder, and Down syndrome. The participants were of both sexes and aged between three and 39 years. All participants who fulfilled the dance protocol were evaluated twice a year. See details in Table 1.

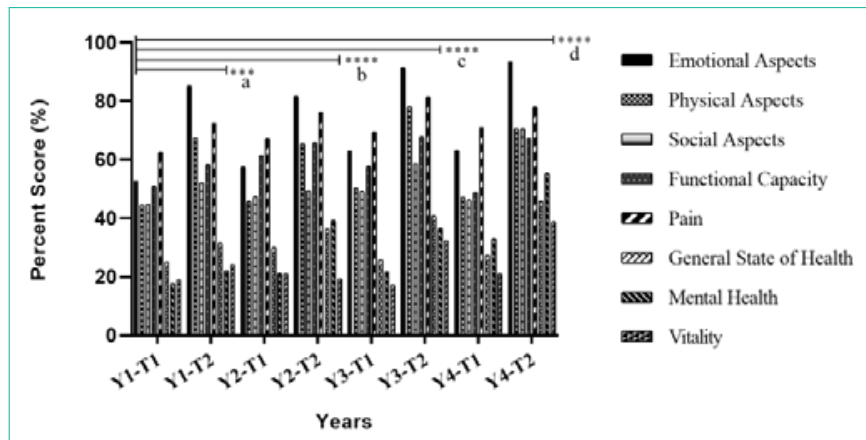

Figure 3: Comparison of the SF-36 score evaluation during four years of application of the dance protocol. The Anderson-Darling normality test $\left(\mathrm{A} 2^{*}\right)$ (norm test $=0.2366$; $p$-value $=0.7784$ ) provides normality test. Two-way analysis of variance (two-way ANOVA $(F(7.49)=19.87 ; \mathrm{P}<0.0001)$ ) compares evolution over the years of evaluation. Dunnett's multiple comparison test shows result obtained by dancing in comparison of the contingency of the variables with year, with a significant result ${ }^{* * *} p=0.007^{* * * * *} p<0.0001$, being: $Y 1$ (year 1) Y2 (year 2) Y3 (year 3), Y4 (year 4), respectively, 2015, 2016, 2017, 2018; T1 evaluation and T2 revaluation each year; a - comparison Y1-T1 and $\mathrm{Y} 1-\mathrm{T} 2$, b-comparison $\mathrm{Y} 1-\mathrm{T} 1$ and $\mathrm{Y} 2$-T2, C-comparison $\mathrm{Y} 1-\mathrm{T} 1$ and $\mathrm{Y} 3-\mathrm{T} 2$, d-comparison $\mathrm{Y} 1-\mathrm{T} 1$ and $\mathrm{Y} 4-\mathrm{T} 2$. 
Table 2: SF-36 values during the 48 months of intervention.

\begin{tabular}{|c|c|c|c|}
\hline \multirow{2}{*}{ Quality of life (SF-36) } & T1 & $\mathrm{T} 2$ & \multirow{2}{*}{$p$-value } \\
\hline & Mean (SEM) & Mean (SEM) & \\
\hline \multicolumn{4}{|l|}{ Functional Capacity } \\
\hline Y1 & $64.91(4.91)$ & $71.71(4.44)$ & $0.0084^{*}$ \\
\hline Y2 & $71.60(4.60)$ & 71.05 (5.08) & 0.4817 \\
\hline Y3 & $64.27(5.05)$ & $71.66(4.81)$ & $0.0267^{\star *}$ \\
\hline Y4 & $58.07(4.45)$ & $71.20(4.44)$ & $0.0003^{* * *}$ \\
\hline \multicolumn{4}{|l|}{ Physical Aspects } \\
\hline Y1 & $50.20(6.34)$ & $72.79(5.66)$ & $0.0112^{\dagger}$ \\
\hline Y2 & $51.51(6.05)$ & $72.65(5.88)$ & $0.0190^{\dagger \dagger}$ \\
\hline Y3 & $51.45(6.28)$ & $80.20(5.13)$ & $<0.0001^{\dagger \dagger+}$ \\
\hline Y4 & $47.99(5.67)$ & $70.61(5.47)$ & $0.0023^{\ddagger}$ \\
\hline \multicolumn{4}{|l|}{ Social Aspects } \\
\hline Y1 & $48.25(4.25)$ & $57.88(3.30)$ & 0.1166 \\
\hline Y2 & $51.09(4.37)$ & $56.32(2.97)$ & 0.3142 \\
\hline Y3 & $50.41(4.10)$ & $60.13(3.34)$ & 0.1123 \\
\hline Y4 & $46.22(3.50)$ & $70.53(4.06)$ & $<0.0001^{\text {执 }}$ \\
\hline \multicolumn{4}{|l|}{ Emotional Aspects } \\
\hline Y1 & $56.74(5.58)$ & $92.10(2.33)$ & $<0.0001^{\text {t+ł }}$ \\
\hline Y2 & $62.00(6.30)$ & $88.13(3.60)$ & $0.0005^{a}$ \\
\hline Y3 & $64.52(6.15)$ & $93.66(2.05)$ & $<0.0001^{b}$ \\
\hline Y4 & $63.25(5.69)$ & $93.49(2.90)$ & $<0.0001^{\mathrm{c}}$ \\
\hline \multicolumn{4}{|l|}{ Vitality } \\
\hline Y1 & $20.47(3.70)$ & 28.99 (4.39) & $0.0196^{d}$ \\
\hline Y2 & $23.27(4.02)$ & 23.09 (3.91) & 0.9067 \\
\hline Y3 & $17.58(3.17)$ & 33.00 (4.99) & $<0.0001^{\mathrm{e}}$ \\
\hline Y4 & $21.00(3.61)$ & $42.42(5.39)$ & $<0.0001^{\dagger}$ \\
\hline \multicolumn{4}{|l|}{ General Health } \\
\hline Y1 & $30.29(3.56)$ & $34.40(4.29)$ & 0.3477 \\
\hline Y2 & $33.24(4.29)$ & $39.53(4.47)$ & 0.1065 \\
\hline Y3 & $26.48(3.72)$ & $41.88(4.85)$ & $0.0017^{g}$ \\
\hline Y4 & $27.37(3.88)$ & 46.88 (5.69) & $0.0009^{h}$ \\
\hline \multicolumn{4}{|l|}{ Pain } \\
\hline Y1 & $69.25(4.44)$ & $82.50(2.51)$ & $0.0006^{i}$ \\
\hline Y2 & 72.50 (3.93) & $81.97(3.01)$ & $0.0093^{j}$ \\
\hline Y3 & $73.04(4.36)$ & $83.50(2.87)$ & $0.0408^{k}$ \\
\hline Y4 & 70.98 (3.65) & 82.07 (3.31) & $0.0159^{\prime}$ \\
\hline \multicolumn{4}{|l|}{ Mental Health } \\
\hline Y1 & $19.51(2.70)$ & $27.82(3.34)$ & $0.0006^{m}$ \\
\hline Y2 & $22.82(3.03)$ & $46.61(4.41)$ & $<0.0001^{n}$ \\
\hline Y3 & 22.39 (3.18) & $39.49(4.60)$ & $<0.0001^{\circ}$ \\
\hline Y4 & $33.02(3.36)$ & $63.02(4.83)$ & $<0.0001^{p}$ \\
\hline \multicolumn{4}{|l|}{ Total } \\
\hline Y1 & 44.56 (1.93) & $58.24(1.33)$ & $<0.0001^{q}$ \\
\hline Y2 & $48.32(2.12)$ & $59.31(1.92)$ & $<0.0001^{r}$ \\
\hline Y3 & 46.27 (1.98) & $62.94(2.12)$ & $<0.0001^{\mathrm{s}}$ \\
\hline Y4 & 45.99 (1.40) & 67.39 (2.41) & $<0.0001^{\mathrm{t}}$ \\
\hline
\end{tabular}

Note: SEM: standard error of mean. Y1 (year 1) Y2 (year 2) Y3 (year 3), Y4 (year 4): 2015, 2016, 2017, 2018, respectively. T1 evaluation and T2 revaluation each year. Wilcoxon test: "Difference between $\mathrm{T} 1$ and $\mathrm{T} 2$ in $\mathrm{Y} 1$ in the functional capacity domain. 95\% Cl. p 0.0084. *Difference between T1 and T2 in Y3 in the functional capacity domain. $95 \% \mathrm{Cl}$. p 0.0267 . ${ }^{* *}$ Difference between T1 and T2 in Y4 in the functional capacity domain. $95 \% \mathrm{Cl}$. p 0.0003 . ${ }^{\dagger}$ Difference between $\mathrm{T} 1$ and T2 in $\mathrm{Y} 1$ in the physical aspects domain. $95 \% \mathrm{Cl}$. p 0.0112. ${ }^{\dagger+}$ Difference between $\mathrm{T} 1$ and $\mathrm{T} 2$ in $\mathrm{Y} 2$ in the physical aspects domain. 95\% Cl. p 0.0190. ${ }^{+\dagger+}$ Difference between $\mathrm{T} 1$ and $\mathrm{T} 2$ in $\mathrm{Y} 3$ in the physical aspects domain. 95\% Cl. $\mathrm{p}<0.0001$. ${ }^{\ddagger}$ Difference between T1 and T2 in Y4 in the physical aspects domain. 95\% Cl. p

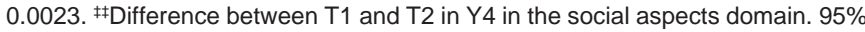

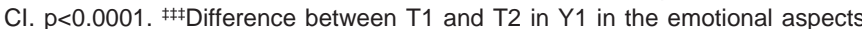

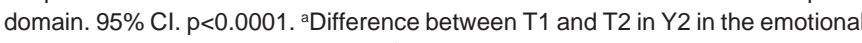
aspects domain. $95 \% \mathrm{Cl}$. p 0.0005. 'D Difference between $\mathrm{T} 1$ and $\mathrm{T} 2$ in $\mathrm{Y} 3$ in the emotional aspects domain. $95 \% \mathrm{Cl}$. $\mathrm{p}<0.0001$. ' ${ }^{\mathrm{D}}$ ifference between T1 and T2 in Y4 in the emotional aspects domain. $95 \% \mathrm{Cl} . \mathrm{p}<0.0001$. dDifference between T1 and $\mathrm{T} 2$ in $\mathrm{Y} 1$ in the vitality domain. $95 \% \mathrm{Cl}$. p 0.0196. eDifference between T1 and $\mathrm{T} 2$ in $\mathrm{Y} 3$ in the vitality domain. $95 \% \mathrm{Cl}$. $\mathrm{p}<0.0001$. 'Difference between T1 and T2 in $\mathrm{Y} 4$ in the vitality domain. $95 \% \mathrm{Cl} . \mathrm{p}<0.0001$. $\mathrm{g}$ Difference between T1 and T2 in $\mathrm{Y} 3$ in the general health domain. 95\% Cl. p 0.0017. $\mathrm{h}$ Difference between T1 and T2 in Y4 in the general health domain. 95\% Cl. p 0.0009. 'Difference between $\mathrm{T} 1$ and $\mathrm{T} 2$ in $\mathrm{Y} 1$ in the pain domain. $95 \% \mathrm{Cl}$. p 0.0006. 'Difference between T1 and $\mathrm{T} 2$ in $\mathrm{Y} 2$ in the pain domain. 95\% Cl. p 0.0093. ${ }^{\mathrm{k}}$ Difference between T1 and $\mathrm{T} 2$ in $\mathrm{Y} 3$ in the pain domain. 95\% Cl. p 0.0408. 'Difference between T1 and T2 in Y4 in the pain domain. 95\% Cl. p 0.0159. mDifference between T1 and T2 in Y1 in the mental health domain. $95 \% \mathrm{Cl}$. p 0.0006. " Difference between T1 and T2 in $\mathrm{Y} 2$ in the mental health domain. $95 \% \mathrm{Cl} . \mathrm{p}<0.0001$. ${ }^{\circ}$ Difference between T1 and $\mathrm{T} 2$ in $\mathrm{Y} 3$ in the mental health domain. 95\% Cl. $\mathrm{p}<0.0001$. ${ }^{\mathrm{P}}$ Difference between $\mathrm{T} 1$ and $\mathrm{T} 2$ in $\mathrm{Y} 4$ in the mental health domain. 95\% Cl. $\mathrm{p}<0.0001$. Paired t test: aDifference between T1 and T2 in $\mathrm{Y} 1$ in total across domains. $95 \% \mathrm{Cl}$. $\mathrm{p}<0.0001$. Wilcoxon test: 'Difference between T1 and T2 in Y2 in total across domains. 95\%

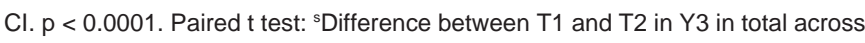
domains. $95 \% \mathrm{Cl}$. $\mathrm{p}<0.0001$. Wilcoxon test: 'Difference between T1 and T2 in Y4 in total across domains. $95 \% \mathrm{Cl}$. $\mathrm{p}<0.0001$.

Table 2 shows the SF-36 values of all participants over the four years of the study, showing the difference between each year in all SF36 domains. The mean values for all domains in the SF-36 increased over the years. In the final year, values increased in all QOL domains.

When the first assessment of the first year (T1-Y1) was compared with the re-assessments of each year (T2-Y1, T2-Y2, T2-Y3, T2-Y4), changes were observed in participants' QOL values in the following domains: physical aspects ( $95 \% \mathrm{CI}, \mathrm{p}=0.0122)$; social aspects $(95 \% \mathrm{CI}$, $\mathrm{p}=0.0008)$; emotional aspects $(95 \% \mathrm{CI}, \mathrm{p}=<0.0001)$; vitality (95\% CI, $\mathrm{p}=<0.0001)$; pain (95\% CI, $\mathrm{p}=0.0289)$; mental health ( $95 \% \mathrm{CI}, \mathrm{p}=<$ $0.0001)$.

Figure 3 shows the results of the percentages of the eight SF-36 domains in each assessment and re-assessment over four years, with the first assessment (Y1-T1) as the basis for comparison. There was a favorable result in the values of all domains in each revaluation: YI-T1 vs. Y1-T2 ( $\mathrm{p}=0.0007), \mathrm{Y} 2-\mathrm{T} 2(\mathrm{p}=0.0001), \mathrm{Y} 3-\mathrm{T} 2(\mathrm{p}=0.0001)$, $\mathrm{Y} 4-\mathrm{T} 2(\mathrm{p}=0.0001)$.

Correlation analysis between SF-36 domains showed the following values: functional capacity and physical aspects $(\mathrm{r}=0.5655$ and $\mathrm{p}=0.0001)$; functional capacity and vitality $(\mathrm{r}=-0.3217$ and $\mathrm{p}=0.0403)$; functional capacity and pain $(\mathrm{r}=0.3396$ and $\mathrm{p}=0.0299)$; physical aspects and general health $(\mathrm{r}=-0.3351$ and $\mathrm{p}=0.0322)$; physical aspects and mental health $(\mathrm{r}=0.4886$ and $\mathrm{p}=0.0012)$; social aspects and vitality $(\mathrm{r}=0.4005$ and $\mathrm{p}=0.0095)$; vitality and general health $(r=0.3962$ and $p=0.0103)$. Table 3 shows all the correlation values between SF-36 domains.

\section{Discussion}

Over four years, 41 participants attended dance classes weekly 
Table 3: Correlations between SF-36 domains quality of life questionnaire.

\begin{tabular}{|c|c|c|c|c|c|c|c|c|c|c|c|c|c|c|c|c|}
\hline & $\begin{array}{l}\text { Functional } \\
\text { cpacity }\end{array}$ & & $\begin{array}{c}\text { Physica1 } \\
\text { aspects }\end{array}$ & & Pain & & $\begin{array}{c}\text { General } \\
\text { Health }\end{array}$ & & Vitality & $\begin{array}{c}\text { Social } \\
\text { aspects }\end{array}$ & & & $\begin{array}{c}\text { Emotional } \\
\text { aspects }\end{array}$ & & $\begin{array}{l}\text { Mental } \\
\text { Health }\end{array}$ & \\
\hline & $r$ & $p$ & $r$ & $p$ & $r$ & $p$ & $r$ & $p$ & $r$ & $p$ & $r$ & $p$ & $r$ & $p$ & $r$ & $p$ \\
\hline $\begin{array}{l}\text { Functional } \\
\text { cpacity }\end{array}$ & & & 0.5655 & 0.0001 & 0.3396 & 0.0299 & --0.2081 & 0.1917 & -0.3217 & 0.0403 & --0.0674 & 0.6753 & 0.0978 & 0.5429 & --0.2302 & 0.1477 \\
\hline $\begin{array}{l}\text { Physica1 } \\
\text { aspects }\end{array}$ & 0.5655 & 0.0001 & & & 0.1279 & 0.4256 & -0.3351 & 0.0322 & --0.2046 & 0.1993 & 0.2846 & 0.0713 & 0.2531 & 0.1104 & |!!.4886 & 0.0012 \\
\hline Pain & 0.3396 & 0.0299 & 0.1279 & 0.4256 & & & --0.0621 & 0.6995 & --0.0990 & 0.5380 & 0.1557 & 0.3311 & 0.2507 & 0.1140 & 0.0002 & 0.9987 \\
\hline $\begin{array}{l}\text { General } \\
\text { Health }\end{array}$ & --0.2081 & 0.1917 & -0.3351 & $0.032 \cdot 2$ & -0.0621 & 0.6995 & & & 0.3962 & 0.0103 & 0.2352 & 0.1388 & -0.1125 & 0.4796 & 0.2404 & 0.1300 \\
\hline Vitality & -0.3217 & 0.0403 & --0.2046 & 0.1993 & -0.0990 & 0.5380 & 0.3962 & 0.0103 & & & 0.4005 & 0.0095 & 0.0361 & 0.8223 & 0.2556 & 0.1968 \\
\hline $\begin{array}{c}\text { Social } \\
\text { aspects }\end{array}$ & --0.0674 & 0.6753 & 0.2846 & O.OJ13 & 0.1557 & 0.3311 & 0.2352 & 0.1388 & 0.4005 & 0.0095 & & & 0.2175 & 0.1720 & 0.0784 & 0.6257 \\
\hline $\begin{array}{c}\text { Emotional } \\
\text { aspects }\end{array}$ & 0.0978 & 0.5429 & 0.2531 & 0.1104 & 0.2507 & 0.1140 & --0.1125 & 0.4796 & 0.0361 & 0.8223 & 0.2175 & 0.1720 & & & 0.2556 & 0.1068 \\
\hline $\begin{array}{l}\text { Mental } \\
\text { Health }\end{array}$ & --0.2302 & 0.1477 & 0.4886 & 0.0012 & 0.0002 & 0.9987 & 0.2404 & 0.1300 & 0.2556 & 0.1968 & 0.0784 & 0.6257 & 0.2556 & 0.1068 & & \\
\hline
\end{tabular}

and, through QOL analysis, several physical and emotional conditions were changed during this period. In the first 10 months of dance practice, there were changes in the values regarding functional capacity, physical and emotional aspects, vitality, pain and mental health. In the second year, after 20 months of dance practice, changes in physical and emotional aspects, pain and mental health impacted the QOL of these participants. In the third year of dance practice, the highlighted domains were: functional capacity, physical and emotional aspects, vitality, general health and mental health. Over 48 months of intervention, all SF-36 domains were influenced by dance practice, mainly social aspects and general health.

It is important to highlight that having a disability does not necessarily affect an individual's QOL [19]. In this view, functional limitations in the lives of PWD usually impair their chances of having a social life; this can affect their mental health and well-being as these are impacted by the restrictions in social relationships and social environments [20,21]. This was highlighted by Perez-Flores et al. [22] in participants with ataxia and by McEvoy et al. [23] in participants with bipolar disorder, depression and schizophrenia. Some studies also highlighted several QOL impairments in different aspects such as physical, functional and social, including pain and mental health and that there is a need for interventions to minimize them in PWD [24-27]. Thus, interventions should be proposed that address QOL in PWD to develop feelings of belonging. The human being itself has a need to belong, be involved and be motivated, and can be negatively affected in terms of mental health and QOL by social exclusion, lack of autonomy and social reward difficulties [28]. Thus, social participation plays a role in generating positive emotions and strengthening relationships [29]. Fekete et al. [28] also showed that it is an important determinant for mental health and QOL, especially in people with disabilities [29] who have various constant difficulties in their daily lives. Stigma surrounding the "perfect body" in daily life and in social media is one of the factors that leads to prejudice against and low self-esteem in PWD, negatively influencing their QOL. In this way, approaches that encourage contact with family, friends and inclusion in socio-emotional practices have been shown to be highly beneficial in contributing to recognition of their own body dialogue, acceptance, self-esteem, and emotional and mental health $[6,9,30]$.

Moreover, people with disabilities face negative effects caused by loneliness. This can affect their social cognition, mood, relationships and social inclusion. The accumulation of these feelings can cause a physiological response to stress and an appearance of depressive symptoms [31]. Thus, it is essential to involve PWD in activities that encourage physical, mental, emotional, cognitive and social wellbeing. The quality of life of PWD is also impaired due to the existing barriers to access and social participation and the lack of services that focus on social participation, a fact that amplifies impairments in theses domains [29]. In this respect, offering approaches and strategies that promote physical and social aspects, such as dance practice, can involve PWD in their environment and encourage mental, emotional, social, health and a better quality of life. According to the WHO guidelines on physical activity and sedentary behavior (2020) [32], physical activity has benefits for physical fitness, cardiorespiratory, cardiometabolic, mental and cognitive health, among others. In addition, it reports the importance of practicing physical exercise that is pleasant, has variety and is appropriate for age and ability. With that in mind, we offer dance as an intervention for the population included in the study.

In this regard, participants in this study showed changes in QOL domains after 48 months (4 years) of dance practice, mainly physical and emotional aspects, pain and mental health. Physical aspects are related to functional capacity with regard to the performance of daily living activities, influencing functional performance [33-35]. TeixeiraMachado and DeSantana, 2013 [6] observed that dance practice could improve physical performance, motor control and reduce anxiety in PWD. The review by Pereira et al., 2018 [36], involving participants with Parkinson's disease, included studies that described how dance practice could influence the motor aspects related to balance, gait and motor control. Thus, dance practice can increase body awareness through body movement based on Laban's (1978) [15] principles (space, fluence, height and time) that underlie attention, spontaneity and action. In the studies by Kwak (2007), Chaitown et al. (2010), Kim et al. (2011), Kim et al. (2012), Shin et al. (2015) and LópezOrtiz, Egan and Gaeber-Spira (2016) [37-42] it was observed that dance practice gave benefits to its practitioner in terms of motor aspects, as well as being positive and enriching for emotional aspects and the mental health of people with cerebral palsy [43].

Regarding emotional aspects and mental health, the review by Lahart et al. (2019) [44] shows how physical activity performed in natural environments can benefit mental health and well-being in those who practice it and how it influences emotional aspects such as anxiety, tension, worry, depression (including bad mood and sadness), providing positive emotions such as self-esteem, joy, tranquility and gratitude. In addition, these practices showed positive 
changes in social interaction, attentional state and memory [44]. During these four years, in addition to dance classes, participants performed in theaters, convention centers, public squares, and other natural spaces. Participants in this feasible study showed changes in emotional aspects and mental health, as well as in interpersonal relationships with other participants. The support of family, friends and other members of this study was vital for these changes seen in participants. In each class they managed to overcome their own obstacles such as aggressiveness, anxiety, communication, depression, difficulty concentrating, insecurity, shyness and social interaction. Over the months, they were able to feel this support, as well as that from their community during public presentations, thus bringing about emotions in the participants such as joy, self-esteem, self-confidence and social reciprocity with members of the group and the audience that attended their performances.

According to a longitudinal study by Ludqvist et al. (2018) [45], most PWD have a greater requirement for health and social assistance resources, are susceptible to the risk of social isolation and face greater restrictions in their lives. Thus, it is important to use strategies, such as dance practice, that provide physical and emotional activities for these people to expand their independence and reduce health and/ or social assistance services. Fekete et al. (2019) [28] highlight how involvement in activities like dance can make a positive impact on basic needs that influence mental health and QOL as they convey the feeling of recognition and admiration from other people, social affiliation, skills development and maintenance. Using dance as one of these strategies, changes observed by Ludqvist et al. (2018) [45] and Fekete et al. (2019) [28] were also noticeable in the present study and ratified through the reports made by the participants and/or their family members after class and/or public presentations. For example: decreased use of drugs and medications, improved communication and functional independence, increased self-esteem and reduced moments of aggression can show how dance influences the physical and mental well-being of those who practice it.

Pain, emotional aspects and mental health have an intrinsic relationship. Pain is a disabling factor for even simple tasks, and this difficulty can trigger a feeling of depression or anxiety in the individual which results in worsening health, mental health and relationships with the environment in which the individual lives [46]. Dance practice appears to transform painful sensations into the pleasure of dance performance [47], a fact that may have been enhanced through public presentations and throughout the period of preparation and choreographic assembly [48]. From this, participants can perceive that their functional capacity can surpass limits, directly impacting self-esteem, happiness and enthusiasm, points of reference of the domains of mental health and emotional aspects [43]. Moreover, some researchers have elucidated that dance can influence psychosocial factors, in addition to reducing social isolation and loneliness, since, during classes, participants have contact with others $[43,49]$.

It is worth highlighting evidence that shows that dance practice improves QOL in people with disabilities. In a systematic review of individuals with autism, DeJesus et al. (2020) [50] highlighted the ability of dance to provide physical, emotional and cognitive wellbeing, resulting in empathy, facial expression, body awareness, and even behavior and social reciprocity. Dos Santos Delabary et al., (2018)
[8] showed in their systematic review and meta-analysis that dance improves QOL and functional mobility in people with Parkinson's disease. Serrano-Guzman et al. (2016) [51] conducted a study in which they observed that dance improved QOL as well as sleep and blood pressure in middle-aged women. Adam, Ramli \& Shahar (2016) [52] conducted a study with 84 participants with cognitive disorders and observed that dance, in addition to improving QOL, also reduced participants' anxiety and depression. These findings are from studies with an intervention period of less than 12 months [8,51,52].

Based on what was obtained and discussed in this study, it seems that dance practice over a long period can establish an improvement in PWD within the social context as it can stimulate contact with other people in daily life. It can reduce social isolation, loneliness, anxiety and aggressiveness. Dance practice can be a strategy capable of improving self-esteem, self-confidence, physical, emotional and psychosocial well-being.

Some factors limited conduction of the present study, such as: distance of some participants to the place where the dance classes and artistic presentations were performed; lack of attendance in classes (approximately ten participants); lack of a control group to compare the collected data; inability to limit participants to doing just dance as an intervention, since, due to their clinical condition, there is a need for multidisciplinary monitoring. Another limitation was the fact that there was a heterogeneous group of participants in relation to clinical conditions. In addition, there is subjectivity in the answers because it is a self-administered questionnaire which was, in most cases, answered by caregivers. Over 48 months, some evaluation data were lost or re-assessment of some participants was not included, which meant they were unable to influence the results of the present study.

\section{Clinical Implications}

Through data obtained in the present study, it is suggested that long-term dance practice can contribute to minimizing social barriers in several aspects for PWD, including physical, mental, emotional and social emphasized in previous studies. Dance is a low-risk and low-cost approach, so it is a regular practice that can be easily used in various populations and spaces, individually or in group. Although not the focus of this study, dance practice may also improve selfesteem and anxiety, since PWD are generally marginalized and/or excluded from society. And through activities such as dance, PWD feel free to express their feelings and emotions. In addition, they can feel the empowerment of being seen as the center of attention for an audience that can watch and honor their dance performance.

\section{Conclusion}

The present study shows that through dance practice over a long period, PWD can improve their QOL in different aspects, mainly in relation to physical aspects, emotional aspects, pain and mental health. Thus, we conclude that over four years, incorporating dance practice into the lifestyle can impact the QOL of PWD, suggesting that it is a practice that contributes to their inclusion in the sociocultural scenario.

\section{Informed Consent}

Informed consent was obtained from all participants and their parents or caregivers included in the study and all data collected 
during the evaluations were anonymous.

\section{Acknowledgment}

The authors thank the Coordenação de Aperfeiçoamento de Pessoal de Nível Superior (CAPES) for funding the research, and all research participants, their families for their trust and contribution to this study and all volunteers during the development of the research. Acknowledgment too Heitor Franco Santos for his collaboration in Figure 3 and Table 4.

\section{Funding}

The study was developed at the Federal University of Sergipe, Brazil. This study was funded by the Coordenação de Aperfeiçoamento de Pessoal de Nível Superior-Brazil (CAPES). Finance code number 400075/2017-2, process number: 23038.011152/2017-62.

\section{Ethical Approval}

All volunteers and parents or caregivers read and signed the informed consent form accepting to participate in the study. The Ethics and Research Committee of Federal University of Sergipe approved this study. All procedures performed in studies involving human participants were in accordance with the ethical standards of the institutional and/or national research committee mentioned above and with the 1964 Helsinki declaration and its later amendments or comparable ethical standards.

\section{References}

1. Vital FMDP, Resende APC. A convencao sobre os direitos das pessoas com deficiencia comentada. Brasília: Secretaria Especial dos Direitos Humanos. Coordenadoria Nacional para Integracao da Pessoa Portadora de Deficiência-CORDE. 2008.

2. Sulaiman SK, Aldersey HM, Fayed N, Kaka B, Okyere C. Quality of life assessment scales in polio survivors: a scoping review. Quality of Life Research. 2019; 28: 2341-2357.

3. World Health Organization (WHO). Word health organization quality of life assessment (WHOQOL): position paper from the Word Health Organization. Social Science \& medicine. 1995; 41: 1403-1409.

4. Pereira EF, Teixeira CS, dos Santos A. Qualidade de vida: abordagens, conceitos e avaliacao. Rev. Bras. Educ. Fis Esp. 2012; 26: 241-250.

5. Yu PA, Hsu WH, Hsu WB, Kuo LT, Lin ZR, Shen WJ, et al. The effects of high impact exercise intervention on bone mineral density, physical fitness, and quality of life in postmenopausal women with osteopenia: A retrospective cohort study. Medicine. 2019; 98: e14898.

6. Teixeira-Machado L, DeSantana J. Dancaterapia e a qualidade de vida de pessoas com deficiência física: ensaio clínico controlado. Rev Bras Qual Vida. 2013; 5: 39-52.

7. McNeely ME, Duncan RP, Earhart GM. Impacts of dance on non-motor symptoms, participation, and quality of life in Parkinson disease and healthy older adults. Maturitas. 2015; 82: 336-341.

8. dos Santos Delabary M, Komeroski IG, Monteiro EP, Costa RR, Haas AN Effects of dance practice on functional mobility, motor symptoms and quality of life in people with Parkinson's disease: a systematic review with metaanalysis. Aging Clin Exp Res. 2018; 30: 727-735.

9. Lopez-Ortiz C, Gaebler-Spira DJ, Mckeeman SN, Mcnish RN, Green D. Dance and rehabilitation in cerebral palsy: a systematic search and review. Dev Med Child Neurol. 2019; 61: 393-398.

10. Murillo-Garcia A, Villafaina S, Adsuar JC, Gusi N, Collado-Mateo D. Effects of dance on pain in patients with fibromyalgia: A systematic review and metaanalysis. Evidence-based Complementary and Alternative Medicine. 2018.

11. Ren J, Xia J. Dance therapy for schizophrenia. Cochrane Database Syst Rev.

\section{3; CD006868}

12. Teixeira-Machado L, DeSantana J. Effect of dance on lower-limb range of motion in young people with cerebral palsy: a blinded randomized controlled clinical trial. Adolesc Health Med Ther. 2019; 10: 21-28.

13. Baars BJ, Franklin S, Ramsoy TZ. Global workspace dynamics: Cortical "binding and propagation" enables conscious contents. Front. Psychol. 2013; 4: 200

14. Sakurada T, Ito K, Gomi H. Bimanual motor coordination controlled by cooperative interactions in intrinsic and extrinsic coordinates. Eur J Neurosci. 2016; 43: 120-130.

15. Laban R. Dominio do movimento. ed. Organized by Lisa Ullmann. Translation of de Anna Maria Barros de Vecchi e Maria Silva Mourao Netto. Sao Paulo: Summus. 1978.

16. Teixeira-Machado L, Araujo FM, Cunha FA, Menezes M, Menezes T, DeSantana JM. Feldenkrais method-based exercise improves quality of life in individuals with Parkinson's disease: a controlled, randomized clinical trial. Altern Ther Health Med. 2015; 21: 8-14.

17. Ciconelli RM, Ferraz MB, Santos W, Meinao I, Quaresma MR. BrazilianPortuguese version of the SF-36. A reliable and valid quality of life outcome measure. Rev Bras Reumatol. 1999; 39: 143-150.

18. Lopez-Ortiz C, Gladden K, Deon L, Schmidt J, Girolami G, Gaebler-Spira D. Dance program for physical rehabilitation and participation in children with cerebral palsy. Arts Health. 2012; 4: 39-54.

19. Hall SS, MacMichael J, Turner A, Mills DS. A survey of the impact of owning a service dog on quality of life for individuals with physical and hearing disability: a pilot study. Health Qual. Life Outcomes. 2017; 15: 59.

20. Tough H, Siegrist J, Fekete C. Social relationships, mental health and wellbeing in physical disability: a systematic review. BMC Public Health. 2017; 17: 414.

21. Hamrin EK, Gustafsson G, Jaracz K. Quality of life among the elderly with locomotor disabilities in Sweden and Poland in the 1990s. Qual Life Res. 2012; 21: 281-289.

22. Perez-Flores J, Hernandez-Torres A, Monton F, Nieto A. Health-related quality of life and depressive symptoms in Friedreich ataxia. Qual Life Res. 2020; 29: 413-420.

23. McEvoy J, Gandhi SK, Rizio AA, Maher S, Kosinski M, Bjorner JB, et al. Effect of tardive dyskinesia on quality of life in patients with bipolar disorder, major depressive disorder, and schizophrenia. Qual Life Res. 2019; 28: 3303-3312.

24. Law M, Hanna S, Anaby D, Kertoy M, King G, Xu L. Health-related quality of life of children with physical disabilities: A longitudinal study. BMC Pediatr. 2014; $14: 26$.

25. Domellof E, Hedlund L, Odman P. Health-related quality of life of children and adolescents with functional disabilities in a northern Swedish county. Qual Life Res. 2014; 23: 1877-1882.

26. Ji J, Zhang L, Zhang Q, Yin R, Fu T, Li L, et al. Functional disability associated with disease and quality-of-life parameters in Chinese patients with rheumatoid arthritis. Health Qual Life Outcomes. 2017; 15: 89.

27. Avila CC, Lima MB, de Azevedo Barros MB. Influence of Diabetes complications and limitations on health-related quality of life: a study in a southeastern Brazilian city. Qual Life Res. 2020; 29: 473-482.

28. Fekete C, Siegrist J, Post MWM, Brinkhof MWG, Swi SCI Study Group. Productive activities, mental health and quality of life in disability: exploring the role enhancement and the role strain hypotheses. BMC Psychol. 2019; 7: 1 .

29. Jespersen LN, Michelsen SI, Tjørnhoj-Thomsen T, Svensson MK, Holstein $\mathrm{BE}$, Due P. Living with a disability: a qualitative study of associations between social relations, social participation and quality of life. Disabil. Rehabil. 2019; 41: $1275-1286$.

30. Shea M. The Quality of Life is Not Strained: Disability, Human Nature, WellBeing, and Relationships. Kennedy Inst. Ethics J. 2019; 29: 333-366. 
31. Tough H, Brinkhof MWG, Siegrist J, Fekete C. The impact of loneliness and relationship quality on life satisfaction: A longitudinal dyadic analysis in persons with physical disabilities and their partners. J Psychosom. Res. 2018; 110: 61-67.

32. WHO guidelines on physical activity and sedentary behaviour. Geneva: World Health Organization; Licence: CC BY-NC-SA 3.0 IGO. 2020.

33. Efraimidou V, Sidiropoulou M, Giagazoglou P, Proios M, Tsimaras V Orologas A. The effect of a music and movement program on gait, balance and psychological parametres of adults with cerebral palsy. Int J Spec Educ. 2016; 31.

34. Green D, Ziviani J. The arts and children's occupational opportunities Occupation-Centered Practice with Children: A Practical Guide for Occupational Therapists, $2^{\text {nd }}$ edition. West Sussex, UK: Wiley-Blackwell. 2017.

35. Howarth L. Creative health: the arts for health and wellbeing. Perspect Public Health. 2018; 138: 26-27.

36. Pereira APS, Marinho V, Gupta D, Magalhaes F, Ayres C, Teixeira S. Music Therapy and Dance as Gait Rehabilitation in Patients With Parkinson Disease: A Review of Evidence. J Geriatr Psychiatry Neurol. 2019; 32: 49-56.

37. Kwak EE. Effect of rhythmic auditory stimulation on gait performance in children with spastic cerebral palsy. J Music Ther. 2007; 44: 198-216.

38. Chaitow L, Rogoff T, Mozgala G, Chmelik S, Comeaux Z, Hannon J, et al. Modifying the effects of cerebral palsy: The Gregg Mozgala story. J Bodyw Mov Ther. 2010; 14: 108-118

39. Kim SJ, Kwak EE, Park ES, Lee DS, Kim KJ, Song JE, et al. Changes in gait patterns with rhythmic auditory stimulation in adults with cerebral palsy. Neuro Rehabilitation. 2011; 29: 233-241.

40. Kim SJ, Kwak EE, Park ES, Cho SR. Differential effects of rhythmic auditory stimulation and neurodevelopmental treatment/Bobath on gait patterns in adults with cerebral palsy: A randomized controlled trial. Clin. Rehabil. 2012 26: $904-914$

41. Shin YK, Chong HJ, Kim SJ, Cho SR. Effect of rhythmic auditory stimulation on hemiplegic gait patterns. Yonsei Med J. 2015; 56: 1703-1713.

42. Lopez-Ortiz C, Egan T, Gaebler-Spira DJ. Pilot study of a targeted dance class for physical rehabilitation in children with cerebral palsy. SAGE open med. 2016; 4: 2050312116670926.
43. Teixeira-Machado L, Azevedo-Santos I, DeSantana JM. Dance improves functionality and psychosocial adjustment in cerebral palsy: a randomized controlled clinical trial. Am J Phys Med Rehabil. 2017; 96: 424-429.

44. Lahart I, Darcy P, Gidlow C, Calogiuri G. The effects of green exercise on physical and mental wellbeing: a systematic review. Int J Environ Res Public Health. 2019; 16: 1352.

45. Lundqvist M, Levin L, Roback K, Alwin J. The impact of service and hearing dogs on health-related quality of life and activity level: a swedish longitudinal intervention study. BMC Health Serv Res. 2018; 18: 497.

46. Kohrt BA, Griffith JL, Patel V. Chronic pain and mental health: integrated solutions for global problems. Pain. 2018; 159: S85-S90.

47. Geneen LJ, Moore RA, Clarke C, Martin D, Colvin LA, Smith BH. Physical activity and exercise for chronic pain in adults: an overview of Cochrane Reviews. Cochrane Database Syst. Rev. 2017; 1: CD011279.

48. Souza-Santos C, Dos Santos JF, Azevedo-Santos I, Teixeira-Machado L. Dance and equine-assisted therapy in autism spectrum disorder: Crossover randomized clinical trial. Clinical Neuropsychiatry. 2018; 15: 284-290.

49. Teixeira-Machado L, Arida RM, de Jesus Mari J. Dance for neuroplasticity: A descriptive systematic review. Neurosci Biobehav Rev. 2019; 96: 232-240.

50. DeJesus BM, Oliveira RC, de Carvalho FO, de Jesus Mari J, Arida RM, Teixeira-Machado L. Dance promotes positive benefits for negative symptoms in Autism Spectrum Disorder (ASD): A systematic review. Complement Ther Med. 2020; 49: 102299.

51. Serrano-Guzman M, Valenza-Pena CM, Serrano-Guzman C, AguilarFerrandiz E, Valenza-Demet G, Villaverde-Gutierrez C. Efectos de un programa de terapia de baile en la calidad de vida, el sueno y la presión arterial en mujeres de mediana edad: un ensayo controlado aleatorizado [Effects of a dance therapy programme on quality of life, sleep and blood pressure in middle-aged women: A randomised controlled trial]. Med Clin (Barc). 2016; 147: 334-339.

52. Adam D, Ramli A, Shahar S. Effectiveness of a combined dance and relaxation intervention on reducing anxiety and depression and improving quality of life among the cognitively impaired elderly. Sultan Qaboos Univ. Med J. 2016; 16: e47-e53. 